ESTUDIOS

\title{
La mediación procesal de conflictos colectivos en la justicia del trabajo brasileña
}

\author{
The procedural mediation of collective conflicts in the brazilian labor court
}

\author{
Fabiana Marion Spengler \\ Universidade de Santa Cruz do Sul, Brasil \\ Márcio Dutra Da Costa \\ Universidade de Santa Cruz do Sul, Brasil
}

\begin{abstract}
RESUMEN El objetivo de este trabajo es discutir el desarrollo de la mediación procesal como método de tratamiento de conflictos colectivos en la justicia del trabajo brasileña. El método de abordaje utilizado es el deductivo, y el método de procedimiento adoptado es el monográfico. Para el tratamiento de tales conflictos, se concluye que la mediación procesal es un método altamente recomendable, pues busca restablecer la capacidad de diálogo entre las partes. Sin embargo, es menester que los magistrados y servidores que estén dispuestos a oficiar como mediadores obedezcan rigurosamente a los principios y reglas contenidos en el Código de Ética de Conciliadores y Mediadores Judiciales, bajo pena de desvirtuar la finalidad del instituto.
\end{abstract}

PALABRAS CLAVE Conflictos laborales colectivos, justicia del trabajo, mediación.

ABSTRACT The purpose of this paper is to discuss the development of procedural mediation as a method of dealing with collective conflicts in the Brazilian Labor Court. The research method used was the deductive, and the procedure method adopted was the monographic. For the treatment of such conflicts, it is concluded that procedural mediation is a highly recommendable method, because it seeks to reestablish the capacity for dialogue between the parties. However, it is necessary that magistrates and servants who are willing to officiate as mediators strictly obey the principles and rules contained in the Code of Ethics of Judicial Conciliators and Mediators, under penalty of distorting the purpose of the institute.

KEYWORDS Collective labor conflicts, labor court, mediation. 


\section{Introducción}

Las relaciones entre el capital y el trabajo están inevitablemente impregnadas por un estado de tensión, debido al intrínseco antagonismo existente entre los empleadores (naturalmente comprometidos en aumentar sus márgenes de ganancia, como es propio al sistema económico capitalista) y los empleados (que, a su vez, anhelan percibir la mayor remuneración posible a cambio de su fuerza de trabajo, invertida en favor de los primeros). Por regla general, tales vínculos se encuentran en una condición de estabilidad, obtenida a partir de mecanismos que pretenden compensar el desequilibrio natural en las relaciones entre el capital y el trabajo, de los cuales la legislación laboral es un ejemplo fundamental.

Sin embargo, hay momentos en que esa tensión latente estalla en forma de conflicto, el cual puede presentarse de modo individual (involucrando sólo a un trabajador o a varios trabajadores considerados individualmente) o colectivo (abarcando un grupo o incluso la totalidad de los trabajadores de un empresa determinada, relacionado con los intereses generales de ese grupo, con o sin la participación del sindicato representante de la categoría profesional).

En casos extremos, es posible que la insatisfacción de los trabajadores aumente de tal manera que resulte en una huelga, con la posibilidad de infligir severos daños al empleador. Si surgiera un conflicto laboral colectivo, será necesario un adecuado tratamiento, ya sea por la vía autocompositiva o por la vía heterocompositiva.

En la autocomposición, las propias partes conflictivas promueven el tratamiento del conflicto, sin que haya la toma de decisión por un tercero ajeno a ellas; lo que, a su vez, es característico de la heterocomposición.

Entre los diversos métodos autocompositivos de tratamiento de conflictos (como la evitación, la autotutela o la autodefensa, la facilitación, la conciliación y la negociación), merece realce la mediación. La mediación —cuyo concepto se expondrá a continuación- puede darse en la órbita judicial o extrajudicial, así como en la esfera procesal o preprocesal.

En la órbita extrajudicial, tiene relevancia en el ordenamiento jurídico brasileño la actuación de dos organismos públicos como promotores de la mediación de conflictos laborales colectivos: el Ministerio de Economía, que desde 2019 es el responsable por las atribuciones del antiguo Ministerio del Trabajo, extinguido en tal año (en particular, las atribuciones que figuran en el artículo 11 de la Ley 10.192, del 14 de febrero de 2001, y el Decreto 1.572, del 28 de julio de 1995); y la Fiscalía Laboral, en Brasil llamada de «Ministerio Público del Trabajo» (según el artículo 83, IX, de la Ley Complementaria 75, del 20 de mayo de 1993, así como lo dispuesto en la Resolución 157, del 28 de agosto de 2018, del Consejo Superior del Ministerio Público del Trabajo).

En el contexto de la justicia del trabajo brasileña, el instituto de la mediación está 
regulado por la Resolución 174, del 30 de septiembre de 2016, editada por el Consejo Superior de la Justicia del Trabajo (CSJT).

Ante este cuadro, el presente artículo presenta el siguiente problema de investigación: ¿De qué manera se desarrolla el instituto de la mediación procesal de conflictos colectivos en los órganos que componen la justicia del trabajo brasileña?

El tema del estudio consiste en el análisis de las posibilidades y de los límites a la utilización de la mediación procesal como método de tratamiento de conflictos de naturaleza colectiva, en el ámbito de la justicia del trabajo brasileña.

El objetivo general es el de investigar el desarrollo de la adopción de la mediación procesal como método de tratamiento de conflictos colectivos en la justicia del trabajo brasileña, así como los límites que se imponen a tal práctica.

Los objetivos específicos son: abordar los aspectos generales relacionados con los conflictos laborales colectivos; describir la clasificación de los principales métodos autocompositivos de tratamiento de conflictos, destacando la mediación; y verificar cómo se desarrolla la aplicación de la mediación procesal ante la justicia del trabajo brasileña, a la luz de los principios fundamentales que rigen dicho instituto.

La justificación del artículo reside en el incipiente desarrollo de los estudios relacionados a la Política Judicial Nacional de Tratamientos Adecuados de las Disputas de Intereses en el ámbito de la justicia del trabajo brasileña, instituida en septiembre de 2016, por medio de la Resolución CSJT núm. 174.

El método de abordaje utilizado fue el deductivo, el cual parte de la relación entre argumentos generales y argumentos particulares hasta llegar a la conclusión. Por su parte, el método de procedimiento adoptado fue el monográfico, a partir de la lectura de normas y de bibliografía relacionadas al tema en estudio.

\section{Conflictos laborales colectivos}

En este capítulo se abordarán algunos aspectos fundamentales de los conflictos laborales colectivos. Inicialmente, se tratará del concepto de conflicto; luego, se pasará al análisis de los conflictos laborales, enfatizando en los que tienen un carácter colectivo.

Etimológicamente, la palabra conflicto es originaria del latín conflictus, participio pasado del verbo confligere, lo cual significa "chocar, estar en desacuerdo». Los elementos con («junto») y fligere («golpear, atacar») están presentes en el proceso de formación del sustantivo.

En el vocabulario jurídico, conflicto tiene el sentido de choque de ideas o de intereses, por lo que se deriva el embate o la divergencia entre hechos, cosas o personas (Silva, 2009: 344).

En la doctrina sociológica es posible encontrar el siguiente concepto de conflicto: 
El conflicto consiste en el choque o la colisión intencional entre dos seres o grupos de la misma especie que manifiestan una intención hostil entre sí, generalmente en relación con un derecho, y que para mantener, afirmar o restaurar ese derecho, intentan romper la resistencia del otro, eventualmente con el recurso a la violencia, que en algunos casos puede tender a la aniquilación física del otro (Freund, 1995: 156: la traducción es nuestra, como todas las siguen). ${ }^{1}$

No obstante, el conflicto se considera una divergencia que fragmenta la armonía y el equilibrio que constituyen el estado regular de la sociedad; no debe ser visto sólo bajo un sesgo negativo, como una patología social:

El conflicto es inevitable y saludable (especialmente si queremos llamar democrática a la sociedad en la que se inserta); lo importante es encontrar medios autónomos de manejarlo huyendo de la idea de que sea un fenómeno patológico y encarándolo como un hecho, un evento fisiológico importante, positivo o negativo conforme los valores insertados en el contexto social analizado. Una sociedad sin conflictos es estática (Spengler, 2016: 112). ${ }^{2}$

En el campo laboral, los conflictos pueden tener naturaleza individual o colectiva, clasificación que no se da simplemente tomando como base la cantidad de trabajadores involucrados, sino el alcance de la materia en discusión. En el primer caso, el conflicto implica un trabajador o varios trabajadores considerados individualmente y el empleador, y se basa en el contrato individual de trabajo (Nascimento y Nascimento, 2018: 531). En el segundo caso - que interesa más directamente al presente estudio-, la caracterización se da debido a sus sujetos, que serán los grupos de trabajadores abstractamente considerados, por un lado, y el grupo de empleadores, por otro lado, con el fin de tratar materias de orden general del grupo (Nascimento y Nascimento, 2018: 531).

De modo general, los conflictos colectivos son conflictos de intereses: los empresarios desean mayores ventajas económicas, mientras que los trabajadores quieren obtener condiciones laborales dignas (Almeida, 2016: 53). Sin embargo, hay otros matices que no pueden ser desconsiderados:

1. «Il conflitto consiste nello scontro o nell'urto intenzionale tra due esseri o gruppi della stessa specie che manifestano un'intenzione ostile l'uno nei confronti dell'altro, generalmente a proposito di un diritto, e che per mantenere, affermare o ristabilire quel diritto cercano di infrangere la resistenza dell'altro, eventualmente con il ricorso alla violenza, la quale può in certi casi tendere all'annientamento físico dell'altro» (Freund, 1995: 156).

2. «O conflito é inevitável e salutar (especialmente se queremos chamar a sociedade na qual se insere de democrática); o importante é encontrar meios autônomos de manejá-lo fugindo da ideia de que seja um fenômeno patológico e encarando-o como um fato, um evento fisiológico importante, positivo ou negativo conforme os valores inseridos no contexto social analisado. Uma sociedade sem conflitos é estática» (Spengler, 2016: 112). 
En efecto, no se trata simplemente de un mero conflicto de intereses, sino que de uno fuertemente reglado por el derecho, en que las partes pretenden y resisten, respectivamente, la asignación de beneficios señalados igualmente con mayor o menor precisión en normas jurídicas; conflicto que, en definitiva, dice relación con el ejercicio de una serie de derechos fundamentales, entre ellos, el derecho a sindicalización y la libertad sindical, por parte de los trabajadores que en tal calidad inician el proceso, y el derecho a la propiedad y a desarrollar cualquier actividad económica, por parte del empleador, todo ello sin perjuicio de otros derechos fundamentales de terceros que puedan verse afectados a consecuencia de la negociación, como ocurre, por ejemplo, durante el curso de una huelga declarada en empresas que prestan servicios básicos para la población, como puede ocurrir con aquellas que ofrecen servicios médicos o educacionales (Fuenzalida Martínez, 2015: 84).

Si no se trata adecuadamente un conflicto laboral colectivo se puede llegar a una situación extrema en la que irrumpe un conocido mecanismo de autotutela: la huelga. En el ordenamiento jurídico brasileño, la huelga es un fenómeno social que se caracteriza por la suspensión colectiva, temporal y pacífica de la prestación de trabajo por los empleados a su empleador. En ese país, el instituto pasó por un continuo proceso de evolución, desde su criminalización inicial, a finales del siglo XIX (por medio del Código Penal de 1890), hasta su declaración como derecho constitucional (inicialmente, por medio de la Constitución de los Estados Unidos de Brasil, del 18 de septiembre de 1946).

Azócar Simonet y Cruz González (2015: 142) sostienen que no cabe duda de que el derecho a la huelga es un «derecho fundamental, cuyo propósito es garantizar una adecuada protección al ejercicio del derecho de organización que gozan los trabajadores, y sobre todo a la posibilidad que finalmente los trabajadores puedan negociar en un plano de igualdad de condiciones con sus empleadores».

Otros ejemplos de consecuencias deletéreas derivadas de conflictos laborales colectivos que no han recibido el tratamiento adecuado son: el boicot (que se caracteriza por la negativa de los trabajadores a prestar servicios a favor de un empleador en particular, lo que conduce a la destrucción del negocio debido a la falta de cooperación), el sabotaje (en el que los trabajadores destruyen o inutilizan la maquinaria del establecimiento, como forma de protesta), el piquete (una forma de presión de los huelguistas sobre los trabajadores que optan por continuar la labor, siendo considerada lícita en el ordenamiento jurídico brasileño, desde que realizada de modo pacífico), la ocupación del establecimiento por los trabajadores (que se niegan a abandonar las instalaciones, así como a prestar servicio), y la huelga de exceso de celo (la cual no debe confundirse con la huelga propiamente dicha; la primera es una operación en que los empleados exageran en la meticulosidad con la que desempeñan el servicio), conforme expone la doctrina (Nascimento y Nascimento, 2018: 555-556).

Otro ejemplo de conflicto laboral colectivo es el que emerge de la dispensa colec- 
tiva (también conocida como «despido masivo»), caracterizada por la rescisión simultánea de los contratos de trabajo de la totalidad o de una parte significativa de los empleados de un empleador. Este acontecimiento supone efectos que extrapolan la esfera individual del trabajador, produciendo graves consecuencias para la localidad en que se sitúa el establecimiento, como el aumento del desempleo y los perjuicios sociales que lo acompañan.

También es posible referir la ocurrencia de conflictos relacionados al incumplimiento patronal de derechos e intereses de los obreros que se caractericen como difusos o colectivos en sentido estricto, cuya definición jurídica es dada por el artículo 81, párrafo único, I y II, del Código de Protección al Consumidor, instituido por la Ley 8.078, del 11 de septiembre de 1990. Estos derechos pueden ser tutelados por medio de una acción civil pública, a ser presentada por alguno de los legitimados activos enumerados en el artículo 5 da Ley 7.347, del 24 de julio de 1985 (como el Ministerio Público del Trabajo o el sindicato representante de la categoría profesional).

En la sección siguiente se abordará la clasificación de los principales métodos de tratamiento de conflictos, con énfasis en el mecanismo autocompositivo y no adversarial denominado mediación.

\section{Métodos de tratamiento de conflictos}

Los métodos de tratamiento de los conflictos pueden catalogarse en dos grupos principales: la autocomposición y la heterocomposición. En el primer grupo, el tratamiento del conflicto es consecuencia de la decisión de los propios actores involucrados (como ejemplos pueden ser citados la evitación, la autotutela o autodefensa, la facilitación, la conciliación, la mediación y la negociación). En el segundo grupo, el tratamiento del conflicto proviene de la decisión de un tercero (como ejemplos está el arbitraje y la jurisdicción) (Arlé, 2016: 134-144).

Otra forma de clasificación de los métodos de tratamiento de los conflictos los distingue en métodos adversariales y no adversariales. En el primer grupo, los que se encuentran en conflicto se enfrentan, y la victoria de uno representa la derrota del otro (la autotutela, el arbitraje y la jurisdicción pueden ser referidos como ejemplos). En el segundo grupo, los actores involucrados en el conflicto no llegan al punto de enfrentarse, pero tienen la intención de lograr un resultado en el que todos puedan ganar. Son ejemplos de métodos no adversariales la negociación, la mediación, la evitación, la facilitación y la conciliación (Arlé, 2016: 134-144).

En un estudio dirigido al instituto de la conciliación, Morales (2011: 205) enseña que la jurisdicción «cada día disminuye su radio de acción, cediéndoselo a la conciliación, cuyos objetivos se logran cada día más y mejor en beneficio de las controversias que obtienen en esta forma mayores satisfacciones en sus resultados y paz y tranquilidad en el ámbito social». 
Entre los métodos autocompositivos y no adversariales de tratamiento de conflictos merece destacarse la mediación.

\section{Mediación}

El término mediación proviene del latín mediare, verbo que significa mediar, intervenir, dividir por la mitad. De tal verbo también viene la expresión mediatione (Spengler, 2017: 20):

De hecho, el espacio de la mediación está, ante todo, en el medio, entre dos extremos. Coincide con su relación y con su existencia. Comparten las distancias y los acercamientos. Antes de ser «medio» era, en el mundo antiguo, mesotes: espacio y virtud al mismo tiempo. Era un estar en el medio y, luego, un asumir el problema, no lejos de rechazar el idios (del que idiota), que cierra a los individuos en el egoísmo vulgar desde su punto de vista privado; privado justamente en el sentido de carente de algo. Indica, sobre todo, un espacio común, participativo, que pertenece también a los extremos entre los cuales se define, incluso los más antagónicos y conflictivos; virtud distante de la abstracción de una terzietà [neutralidad] y de una imparcialidad solamente imaginarias (Spengler y Spengler Neto, 2008: 106; cursivas en el original). ${ }^{3}$

La mediación es un proceso informal y flexible, donde se inserta la figura de un tercero imparcial que actúa con la finalidad de ayudar a las partes a recomponer y administrar un conflicto (Felipe y Pinto, 2018: 158).

Según Pereira, la mediación «surge como vía alternativa para dar voz a las partes y estimular el diálogo entre los diversos actores sociales. Es, así, una forma de escucha y pacificación social, respaldada en el reconocimiento y en el respeto a las diferencias» (2016: 17). ${ }^{4}$

Como nota distintiva de otros métodos de tratamiento de conflictos, el diferencial de la mediación consiste en promover la integración entre los litigantes en busca de

3. «De fato, o espaço da mediação está, antes de tudo, no meio, entre dois extremos. Coincide com sua relação e com sua existência. Compartilham as distâncias e os avizinhamentos. Antes de ser «meio» era, no mundo antigo, mesotes: espaço e virtude ao mesmo tempo. Era um estar no meio e, então, um assumir o problema, não distante de recusar o idios (do qual idiota), que fecha os indivíduos no egoísmo vulgar do seu ponto de vista privado; privado justamente no sentido de carente de alguma coisa. Indica, sobretudo, um espaço comum, participativo, que pertence também aos extremos entre os quais se define, mesmo os mais antagônicos e conflitantes; virtude distante da abstração de um terzieta e de uma imparcialidade somente imaginárias» (Spengler y Spengler Neto, 2008: 106).

4. «Surge como via alternativa para dar voz às partes e estimular o diálogo entre os diversos atores sociais. É, assim, uma forma de escuta e pacificação social, lastreada no reconhecimento e no respeito às diferenças» (Pereira, 2016: 17). 
la solución de su conflicto, en comparación con las soluciones impositivas que caracterizan los modelos no consensuados (Pinho y Ramalho, 2017: 5).

Muñoz registra que «la realidad se impone y hay una necesidad imperiosa de disminuir la litigiosidad ante los tribunales de trabajo y de desjudicializar en lo posible el número de procesos laborales de carácter individual» (2018: 186).

En esta segunda década del siglo XXI, el Estado brasileño ha sido bastante prolífico en el ejercicio de la elaboración de leyes relativas a los métodos autocompositivos de tratamiento de conflictos.

El 29 de noviembre de 2010, el Consejo Nacional de Justicia (CNJ) publicó la Resolución núm. 125. Dicha resolución trata sobre la Política Judicial Nacional de tratamiento adecuado de los conflictos de intereses en el ámbito del Poder Judicial, y ha sido inspirada por diversos factores, como: concretar los objetivos estratégicos de la eficiencia operacional, del acceso al sistema de Justicia y de la responsabilidad social, previstos en el artículo 1, IV, a, b y c, de la Resolución CNJ núm. 70/2009 (posteriormente revocada por el artículo 17 de la Resolución CNJ núm. 198/2014); el acceso al ordenamiento jurídico justo, corolario del derecho de acceso a la justicia, consagrado en el artículo 5, XXXV, de la Constitución de la República Federativa del Brasil, de 5 de octubre de 1988 (CRFB); la amplia y creciente escala de la ocurrencia de problemas jurídicos y de conflictos de intereses, lo que exigió la adopción de una política pública de tratamiento adecuado de tales cuestiones; la necesidad de consolidar una política pública permanente de estímulo y perfeccionamiento de los mecanismos consensuados de solución de litigios; la reducción de la excesiva judicialización de los conflictos de intereses, de la cantidad de recursos y de ejecución de sentencias propiciada por la conciliación y por la mediación, reconocidas como instrumentos efectivos de pacificación social, solución y prevención de litigios; y la pertinencia y la necesidad de organizar y uniformizar los servicios de conciliación, mediación y otros métodos consensuados de solución de conflictos, con el fin de evitar disparidades de orientación y prácticas, así como para garantizar la correcta ejecución de la política pública, respetando las especificidades de cada segmento de la justicia. A través de la Enmienda núm. 2, del 8 de marzo de 2016, el CNJ decidió por la publicación de una resolución específica sobre la Política Judicial de tratamiento adecuado de los conflictos de intereses en la Justicia del Trabajo, conforme el artículo 18-B de la Resolución CNJ núm. 125/2010.

El 16 de marzo de 2015 se promulgó la Ley 13.105, la cual entró en vigor el 18 de marzo de 2016 e instituyó el nuevo Código de Proceso Civil (CPC), sucesor de la Ley 5.869/1973. La Sección V del Capítulo III (titulada «De los Auxiliares de la Justicia») del Título IV («Del Juez y de los Auxiliares de la Justicia») del Libro III («De los Sujetos del Proceso») se refiere a «Los Conciliadores y Mediadores Judiciales», más específicamente en los artículos 165 a 175. Según el artículo 769 de la Consolidación de las Leyes del Trabajo (CLT) -aprobada por el Decreto-Ley 5.452, del 1 de mayo de 
1943-, el CPC tiene aplicación subsidiaria al derecho procesal laboral, en casos de omisión en la ley laboral y de compatibilidad con las normas del Título X de la CLT (que dispone sobre el procedimiento judicial laboral).

Poco tiempo después, el 26 de junio de 2015, fue sancionada la Ley 13.140, con el objetivo principal de disponer sobre la mediación entre particulares como medio de solución de controversias y sobre la autocomposición de conflictos en el ámbito de la administración pública. Sin embargo, el párrafo único del artículo 42 de esa ley - también conocida en Brasil como «Marco Legal de la Mediación»- excluye expresamente la aplicación a las relaciones de trabajo de sus dispositivos referentes a la mediación, remetiendo tal reglamentación a una legislación propia.

El 30 de septiembre de 2016, el CSJT —órgano que funciona junto al Tribunal Superior del Trabajo (TST) y es el responsable del ejercicio, en la forma de la ley, de la supervisión administrativa, presupuestaria, financiera y patrimonial de la Justicia del Trabajo de primer y segundo grados en Brasil (CRFB, artículo 111-A, $\$ 2^{\circ}$, II)publicó la Resolución núm. 174, en atención a lo dispuesto en el artículo 18-B de la Resolución CNJ núm. 125/2010. De las razones que condujeron a la edición de tal certificado, además de las referidas arriba en los consideranda de la Resolución CNJ 125/2010, es posible destacar: la existencia del Acto Conjunto TST CSJT GP 9/2016, el cual instituyó la Comisión Nacional de Promoción a la Conciliación, como manifestación de la valorización de la conciliación como política pública judicial; y la valorización de las soluciones conciliatorias como forma de entrega de la prestación jurisdiccional, prevista en el artículo 764 de la CLT (según el cual las disputas individuales o colectivas sometidas a la apreciación de la justicia del trabajo están siempre sujetas a la conciliación, siendo el deber de los magistrados de esta rama especializada del Poder Judicial emplear siempre sus buenos oficios y persuasión hacia una solución conciliatoria de los conflictos, según el caput y el $\$ 1$ de dicho artículo).

En la sección siguiente se abordará la adopción del instituto de la mediación procesal en la justicia del trabajo brasileña a la luz de las normas aplicables a la especie, a saber: el CPC y la Resolución CSJT 174/2016.

\section{Mediación procesal en la Justicia del Trabajo brasileña}

El artículo 1, II, de la Resolución CSJT 174/2016 define la mediación de la siguiente manera:

'Mediación' es el medio alternativo de resolución de disputas en que las partes confían a una tercera persona -magistrado o servidor público por éste siempre supervisado-, la función de aproximarlas, empoderarlas y orientarlas en la construcción de un acuerdo cuando el litigio ya está instaurado, sin la creación o propuesta de opciones para la composición del litigio. 
Inicialmente, llama la atención el hecho de que esa resolución considere la mediación como un medio alternativo de resolución de «disputas», cabiendo el registro de que el término disputa es por ella definido como «la suma de la cuestión jurídica y del conflicto, así considerada a partir de la judicialización del litigio» (según el artículo $1, \mathrm{~V})$.

No obstante el término resolución sea adoptado por la mayor parte de la doctrina brasileña, se entiende que ese vocablo no es el más apropiado, y es preferible la expresión «tratamiento de conflictos»:

Mucho se habla de solución (o resolución) del conflicto. En realidad, sin embargo, no siempre es posible que sea resuelto (en el sentido de ser extinto) por un acto aislado; a menudo el impase tiene fases y sólo es efectivamente superado después de una serie de experiencias vividas a lo largo del tiempo por los involucrados. Se destaca aquí la noción ya mencionada de «transformación del conflicto».

Además, en algunas circunstancias los involucrados ni siquiera desean encerrar totalmente la relación, sino sólo componer una específica situación controvertida; como ejemplo, considere el caso de una relación continua en que las partes quieren (o necesitan) mantener el contrato por ser empresas asociadas. En otras situaciones, aunque quisieran, las partes no podrían encerrar totalmente su vínculo por tener vínculos permanentes (por ejemplo, en virtud de parentesco) (Tartuce, 2018: 18). ${ }^{5}$

La interpretación literal del artículo 1, II, de la Resolución CSJT 174/2016 induce a la idea de que la mediación, en el ámbito de la justicia del trabajo, sería exclusivamente desarrollada en la fase procesal, porque las partes a ser mediadas son estimuladas a la construcción de un acuerdo "cuando el litigio ya esté establecido». Sin embargo, el artículo $7, \$ 7$, de la norma no elide la posibilidad de desarrollo de la mediación antes del enjuiciamiento de la acción, al estatuir que los conflictos colectivos pueden ser sometidos al procedimiento de la mediación preprocesal. En el marco del TST, la mediación preprocesal fue disciplinada por el Acto 168/TST GP, del 4 de abril de 2016, publicado unos seis meses antes de la Resolución CSJT 174/2016.

El artículo 769 de la CLT dispone: «En los casos omisos, el derecho procesal común será fuente subsidiaria del derecho procesal del trabajo, excepto en aquello en

5. «Muito se fala em solução (ou resolução) do conflito. Em realidade, porém, nem sempre é possível que ele seja resolvido (no sentido de ser extinto) por um ato isolado; muitas vezes o impasse tem fases e só é efetivamente superado após uma série de experiências vividas ao longo do tempo pelos envolvidos. Sobreleva aqui a já mencionada noção de "transformação do conflito". Ademais, em algumas circunstâncias os envolvidos sequer desejam encerrar totalmente a relação, mas apenas compor uma específica situação controvertida; como exemplo, considere o caso de uma relação contínua em que as partes querem (ou necessitam) manter o contrato por serem empresas parceiras. Em outras situações, ainda que quisessem, as partes não poderiam encerrar de vez seu vínculo por terem ligações permanentes (p. ex., por força de parentesco)» (Tartuce, 2018: 18). 
que sea incompatible con las normas de este Título». Para determinar si los dispositivos que versan sobre mediación en el CPC son aplicables a las acciones que tramitan ante la justicia del trabajo, es necesario analizar los dos requisitos exigidos por el artículo 769 de la CLT, a saber: omisión y compatibilidad.

En cuanto al primer requisito, no queda ninguna duda en considerarlo cumplido, pues la CLT es omisa respecto a la mediación. Por otra parte, el segundo requisito también está debidamente satisfecho. El instituto de la conciliación (el cual, en el CPC, es abordado en la misma sección que la mediación) se confunde con la propia génesis del proceso laboral en Brasil; así, los actuales Juzgados del Trabajo (primer grado o instancia de la jurisdicción laboral, llamados de "Varas do Trabalho») eran antiguamente denominadas «Juntas de Conciliación y Juicio» («Juntas de Conciliação e Julgamento», según los artículos 647 a 667 de CLT). Estos órganos —actualmente extintos por fuerza del artículo 1 de la Enmienda Constitucional 24/1999- poseían composición paritaria, y estaban compuestos por un juez togado (el cual ocupaba el cargo de Presidente de la Junta) y dos vocales (un representante de los empleados y otro de los empleadores), y tenían por atribución conciliar y juzgar las materias arrolladas en la letra a del caput del artículo 652 de la CLT.

Según el CPC (artículo 165, \$\$ 2 y 3 ), hay dos rasgos distintivos principales entre el menester del conciliador y el del mediador. El conciliador actúa, de modo preferencial, en los casos en que no hay vínculo anterior entre las partes. Por ejemplo, es posible citar las relaciones de consumo o los casos de indemnización de daños materiales derivados de accidentes de tránsito. En tales situaciones, las partes a conciliar, por regla general, ni siquiera se conocían antes de la instauración del conflicto, y no tienen interés en establecer ningún vínculo con la otra parte tras el cumplimiento del acuerdo durante el procedimiento de conciliación. Por su parte, el mediador oficia, esencialmente, en los casos en que hay algún vínculo previo entre las partes (como en los conflictos intrafamiliares, por ejemplo). En ese sentido, la doctrina así se manifiesta:

En la conciliación existen conflictos esporádicos, sin relación previa o posterior entre los conflictivos. Por ejemplo: relaciones de consumo o accidentes de tránsito; ya en la mediación existen conflictos en los que los involucrados poseen relaciones cercanas anteriores al litigio y que permanecerán (al menos así se espera), íntegros tras la resolución del mismo. Los ejemplos típicos son los conflictos del derecho de familia, derecho de sucesión o aquellos pertenecientes a las relaciones de amistad y de vecindad (Spengler, 2017: 74). ${ }^{6}$

6. «Na conciliação existem conflitos esporádicos, sem relacionamento prévio ou posterior entre os conflitantes. Ex.: relações de consumo ou acidente de trânsito; já na mediação existem conflitos nos quais os envolvidos possuem relações próximas anteriores ao litígio e que permanecerão (pelo menos assim se espera), íntegros após a resolução do mesmo. Exemplo típico são os conflitos do direito de 
En esa senda, la esperada persistencia de las relaciones contractuales entre el empleador y sus empleados, después del tratamiento del conflicto, hace recomendable la utilización de la mediación en los conflictos laborales colectivos.

Además, según el CPC, existe un segundo rasgo distintivo entre las figuras del conciliador y del mediador: la postura de esos auxiliares de la justicia ante las partes. Mientras el primero llega al punto de sugerir soluciones para el litigio, el segundo intenta reconectar las partes, restableciendo el diálogo perdido, a fin de que ellas mismas hagan esfuerzos para la construcción conjunta de una solución consensuada.

En el punto siguiente, se discurrirá sobre la estructuración de las unidades judiciales para la atención de lo dispuesto en la Política Judicial Nacional de Tratamiento Adecuados de las Disputas de Interés, en el ámbito de la justicia del trabajo brasileña.

\section{Estructuración de los órganos de la justicia del trabajo brasileña para la práctica} de la mediación

El artículo 2, párrafo único, de la Resolución CSJT 174/2016 atribuye a los Tribunales Regionales del Trabajo (TRT), órganos de segundo grado de la jurisdicción laboral brasileña, la incumbencia de instituir un Núcleo Permanente de Métodos Consensuados de Solución de Disputas (NUPEMEC-JT), así como Centros Judiciales de Métodos Consensuados de Solución de Disputas (CEJUSC-JT). La implementación de estos órganos atiende a la directriz de centralización de las estructuras judiciales de solución consensuada de disputas, prevista en el artículo 3, I, de la citada resolución.

El NUPEMEC-JT está constituido por magistrados y servidores activos designados, pudiendo contar con la participación de inactivos, siempre que estos declaren, bajo su responsabilidad personal, que no militan como abogados en el área bajo jurisdicción de los órganos cubiertos por el CEJUSC-JT. Está prohibida la realización de conciliación o mediación judicial, en el ámbito de la justicia del trabajo brasileña, por personas que no pertenezcan a los cuadros de activos o inactivos del respectivo TRT (artículos 5, caput, y 6, $\$ \$ 6$ y 8, de la Resolución CSJT 174/2016).

Con respecto a esa especie de «reserva de mercado» a profesionales de la justicia del trabajo, la doctrina hace el siguiente cuestionamiento:

En ese sentido, la duda que se instala es acerca de la habilidad de magistrados y servidores (de la activa, o jubilados) de hacer mediación/conciliación, cuyo sesgo y el resultado esperado son tan diferenciados de sus actividades cotidianas. Del magistrado se espera la decisión, la última palabra, y no mediación o conciliación. En la lógica del Poder Judicial, se trabaja con la idea de transferencia de responsabilidades cuanto a la gestión del conflicto que va dirigida al juez que lo traduce en su lenguaje.

família, direito sucessório ou aqueles pertencentes às relações de amizade e vizinhança» (Spengler, 2017: 74). 
Cappelletti y Garth (2002: 159) advierten del peligro de acumular las funciones de decidir (juez) con las funciones de conciliar (conciliador) ya que: «como conciliador puede inconscientemente imponer un 'acuerdo' por la amenaza implícita en su poder de decidir. Como Juez, puede dejar que su esfuerzo de conciliación subvierta su mandato de aplicador de la ley». Si el juez es también mediador, mayor será la preocupación (Spengler, 2017: 125).?

El NUPEMEC-JT tiene varias atribuciones, entre las cuales se destacan las de promover, incentivar y fomentar la investigación, estudios y el perfeccionamiento de los métodos de mediación y conciliación, individuales y colectivos, así como las prácticas de gestión de conflictos; incentivar y promover la capacitación, el entrenamiento y la actualización permanente de magistrados y servidores en los métodos consensuados de solución de conflictos, con foco en el empoderamiento de las partes para la autocomposición de la disputa; e instituir, en conjunto con la Escuela Judicial Regional, cursos de formación inicial, de formación continua y de formación de formadores, todos específicos en las técnicas de conciliación y mediación ante la Justicia del Trabajo (artículo 5, IV, VI y VIII, de la Resolución CSJT 174/2016).

La coordinación del NUPEMEC-JT es ejercida, privativamente, por uno o más magistrados de la activa, indicados por el presidente del respectivo TRT sobre la base de criterios objetivos, pudiendo haber acumulación con la coordinación del CEJUSC-JT, quedando a cargo de la presidencia del TRT el análisis de la conveniencia y oportunidad de la designación exclusiva de magistrados para tales actividades, según el artículo 5, \$2, de la Resolución CSJT 174/2016.

A su vez, el CEJUSC-JT es una unidad vinculada al NUPEMEC-JT y coordinada por un magistrado de la activa, según el artículo 6, caput y $\$ 2$, de la Resolución CSJT 174/2016. El juez coordinador podrá ser auxiliado por jueces supervisores, los cuales, además de supervisar las actividades de los mediadores y conciliadores, son responsables de homologar los acuerdos entablados (artículos 5, \$ 2, y 7, caput, de la Resolución CSJT 174/2016).

En la siguiente sección se abordará el procedimiento de mediación de conflictos laborales colectivos ante los órganos que componen la justicia del trabajo brasileña.

7. «Nesse sentido, a dúvida que se instala então é a respeito da habilidade de magistrados e serventuários (da ativa, ou aposentados) de fazer mediação/conciliação, cujo viés e o resultado esperado são tão diferenciados das suas atividades cotidianas. Do magistrado se espera a decisão, a última palavra, e não mediação ou conciliação. Na lógica do Judiciário, trabalha-se com a ideia de transferência de responsabilidades quanto à gestão do conflito que vai direcionada ao juiz que o traduz na linguagem dele. Cappelletti e Garth (2002, p. 159) que advertem para o perigo de cumular as funções de decidir (juiz) com as funções de conciliar (conciliador) uma vez que: «como conciliador ele pode inconscientemente impor um 'acordo' pela ameaça implícita em seu poder de decidir. Como Juiz, ele pode deixar seu esforço de conciliação subverter seu mandato de aplicador da lei». Se o juiz for também mediador, maior é a preocupação» (Spengler, 2017: 125). 


\section{Procedimiento de mediación procesal en la justicia del trabajo brasileña}

En la Justicia del Trabajo brasileña, las sesiones de mediación son realizadas en el ámbito del CEJUSC-JT, independientemente de la etapa o instancia en que esté tramitando el proceso, aunque haya recurso pendiente en el TST (artículo 6, caput, de la Resolución CSJT 174/2016).

La sala de mediación debe contener una mesa redonda (en un número máximo de seis mesas para cada magistrado supervisor), debiendo preservarse la privacidad de las partes y abogados, según el artículo 7, \$9, de la Resolución CSJT 174/2016.

Durante la realización de las sesiones de mediación, un magistrado debe estar presente en el CEJUSC-JT, quien, además de supervisar la actividad de los mediadores, también puede oficiar en esa condición. El magistrado debe estar siempre a disposición de las partes a ser mediadas y de los abogados, y es indispensable la presencia del abogado de la parte demandante (llamada «reclamante») en los casos de disputas individuales (artículo 6, \$1, de la Resolución CSJT 174/2016).

Una alternativa a la presencia física de las partes es la mediación por medios electrónicos, según autorizado por el artículo 5, IX, de la Resolución CSJT 174/2016.

Como ya se ha expuesto anteriormente, el artículo 1, II, de la Resolución CSJT 174/2016 incumbe al mediador judicial la función de aproximar, empoderar y orientar a las partes en la construcción de un acuerdo cuando la demanda ya está instaurada, sin la creación o propuesta de opciones para la composición del litigio. Sin embargo, tal dispositivo no pasó ileso a la crítica doctrinal:

El inciso en cuestión (II del artículo 3) así como los incisos I y II del artículo 1 dejan claro la posibilidad del mediador/conciliador orientar las partes en la construcción de un acuerdo, algo que no es factible/posible en la actuación del mediador, siendo permitido, sí, al conciliador. Se sabe que no corresponde a ese tercero imparcial decidir por las partes u obligarlas a firmar un acuerdo. Esto se da porque el mediador/ conciliador es un tercero que se coloca entre las partes, no por encima de ellas y que no tiene la prerrogativa de decidir conflictos. Esta prerrogativa se dirige solamente a los magistrados, recordando que mediador/conciliador que sugiere, orienta, decide y obliga a hacer acuerdo es aquel que no conoce su misión y ni su Código de Ética comportándose como un juez, pero en tamaño bonsái (Spengler, 2017: 122-123). ${ }^{8}$

8. «O inciso em questão (II do art. $3^{\circ}$ ) bem como os incisos I e II do art. $1^{\circ}$ deixam claro a possibilidade do mediador/conciliador orientar as partes na construção de um acordo, coisa que não é cabível/possível na atuação do mediador sendo permitido, sim, ao conciliador. Sabe-se que não cabe a esse terceiro imparcial decidir pelas partes ou obriga-las a firmar acordo. Tal se dá porque o mediador/conciliador é um terceiro que se coloca entre as partes, não acima de las e que não possui a prerrogativa de decidir conflitos. Essa prerrogativa é direcionada somente aos magistrados, recordando que mediador/conciliador que sugere, orienta, decide e obriga a fazer acordo é aquele que não conhece sua missão e nem o seu Código de Ética comportando-se como um juiz, porém em tamanho bonsái» (Spengler, 2017: 122-123). 
Con la designación de la sesión de mediación, las partes implicadas en el conflicto laboral colectivo (por regla general, el sindicato representante de la categoría profesional o lo Ministerio Público del Trabajo, en el polo activo, y el sindicato representante de la categoría económica o un empleador, en el polo pasivo) son notificadas a acudir al acto. Esta comparecencia debe ser voluntaria, porque una de las reglas que rigen la mediación es la autonomía de la voluntad, es decir, el «deber de respetar los diferentes puntos de vista de los involucrados, asegurándoles que lleguen a una decisión voluntaria y no coercitiva, con libertad para tomar las propias decisiones durante o al final del proceso y de interrumpirlo en cualquier momento» (artículo 2, II, del Código de Ética de Conciliadores y Mediadores Judiciales, constante en el Anexo II de la Resolución CSJT 174/2016).

En este camino, la doctrina dispone que, aunque la mediación no posea carácter obligatorio, pues solo es voluntaria, llevada a cabo por consenso de los interesados, aun así se muestra como un mecanismo eficaz de tratamiento de conflictos (Gunther, 2016: 46).

No llegando las partes a un acuerdo en la primera sesión, otras sesiones podrán ser designadas, tantas como sean necesarias para lograr la solución consensuada del conflicto (artículo 7, \$5, de la Resolución CSJT 174/2016).

Cabe señalar que una relevante medida impuesta por el artículo 5, II, de la Resolución CSJT 174/2016 fue la prohibición de la imposición, a los magistrados y servidores mediadores, del cumplimiento de metas relacionadas a la cantidad de acuerdos entablados (que parece haber sido olvidada en la Resolución CNJ 125/2010). La idea de medir el éxito de la actuación de un mediador según parámetros numéricos es absolutamente errónea:

Es interesante señalar que una mediación que ha terminado debido a la pérdida del objeto de litigio (reconciliación, por ejemplo) o porque se ha vuelto improductiva (y por lo tanto inviable) no es, necesariamente, una mediación que ha tenido éxito. Cualquier procedimiento de mediación es exitoso si, a pesar de que no existe consenso, las personas puedan retomar el diálogo interrumpido, demostrando, a partir de las sesiones, condiciones de comunicarse de manera más armónica (Spengler, 2017: 183). ${ }^{9}$

Si las partes llegan a un acuerdo, el mediador lo reduce a término, a ser firmado por los presentes y homologado por el magistrado oficiante en el CEJUSC-JT (artícu-

9. «É interessante referir que uma mediação que se encerrou por perda do objeto de litígio (reconciliação, por exemplo) ou porque se tornou improdutiva (e por isso inviável) não é, necessariamente, uma mediação da qual não se obteve êxito. Qualquer procedimento mediativo é exitoso se, não obstante não existir consenso, as pessoas puderem retomar o diálogo interrompido, demonstrando, a partir das sessões, condições de se comunicar de modo mais harmônico» (Spengler, 2017: 183). 
lo 7, caput, de la Resolución CSJT 174/2016). El acuerdo debe respetar siempre el orden público y las leyes vigentes, de acuerdo con el principio fundamental que rige el oficio del mediador (artículo 1, V, del Código de Ética de Conciliadores e Mediadores Judiciales, que figura en el Anexo II de la Resolución CSJT 174/2016). De lo contrario, el magistrado podrá negarse a homologar el acuerdo, no siendo apropiada la impetración de recurso de protección contra esa decisión, según el Precedente 418 de TST.

Si no es posible llegar a un acuerdo y las partes rechazan la propuesta de designación de una nueva sesión de mediación, no puede haber ninguna consecuencia negativa para ellas, debiendo el proceso reanudar su tramitación regular. En este caso, se entiende que el magistrado que ofició de mediador no podrá participar en la instrucción y juicio del acuerdo, pues el artículo 7 del Código de Ética de Conciliadores y Mediadores Judiciales lo declara absolutamente impedido de prestar servicios profesionales, de cualquier naturaleza, a los implicados en el proceso de mediación bajo su conducción (siendo el Código de Ética aplicable a los magistrados y servidores, según el artículo $7, \$ 8$, de la Resolución CSJT 174/2016). Así, el proceso deberá ser redistribuido al magistrado substituto (artículo 656, caput, de la CLT).

\section{Principios de la mediación procesal en la justicia del trabajo brasileña}

Los principios fundamentales que rigen las acciones de mediadores en la justicia del trabajo brasileña están dispuestos en el artículo 1 del Código de Ética de Conciliadores y Mediadores Judiciales, que figura en el Anexo II de la Resolución CSJT 174/2016.

El primer principio es el de la decisión informada, definido como el «deber de mantener el litigante plenamente informado en cuanto a sus derechos y al contexto fático en el que está inserto» (inciso I del artículo 1 del Anexo II). Ese principio está relacionado con la necesidad de proporcionar aclaraciones a los participantes de cómo funciona la sesión, y también sobre la elección de participar o no de la mediación, proponer acuerdos o callarse, aceptarlos o refutarlos (Spengler, 2017: 150).

El segundo principio es el de la competencia, definido como el «deber de [el mediador] poseer cualificación que lo habilite a la actuación judicial, con capacitación en la forma de esta Resolución, observado el reciclaje periódico obligatorio para formación continua» (inciso II del artículo 1). En efecto, el mediador judicial no puede prescindir de una adecuada capacitación para el ejercicio de su menester, siendo la competencia autocompositiva uno de los tópicos que integran el contenido programático mínimo de los cursos de capacitación y perfeccionamiento, que figura en el Anexo I de la Resolución CSJT 174/2016.

El tercer principio es el de la imparcialidad, definido en el inciso III del artículo 1 como el «deber de actuar con ausencia de favoritismo, preferencia o prejuicio, asegurando que valores y conceptos personales no interfieran en el resultado del trabajo, comprendiendo la realidad de los involucrados en la disputa y jamás aceptando cual- 
quier tipo de favor o regalo». Se trata de un principio inherente a la propia magistratura, siendo absolutamente inconcebible que un mediador (el cual, por naturaleza, debe estar posicionado "entre» las partes) pretenda favorecer a una de las partes a ser mediadas, infringiendo la relación de confianza en que debe basarse el procedimiento: «la imparcialidad del mediador radica en la equidistancia frente a las partes mediadas: el mediador no puede aliarse a una de las partes o tomar un partido, a pesar de los previsibles esfuerzos de los mediados en cautivar su preferencia (Lopes y Patrão: 2016: 55). ${ }^{10}$

Según Regla (2015: 123-124), la imparcialidad es un principio deontológico de la mediación, que se proyecta fundamentalmente en dos direcciones: la pretensión de corrección (los acuerdos no pueden violar el derecho no disponible) y la erradicación del abuso y de la mera imposición actoral (es necesario construir un marco de debate aceptable).

El cuarto principio presente es el de la independencia y autonomía, definido como el «deber de actuar con libertad, sin sufrir ninguna presión interna o externa, siendo permitido rechazar, suspender o interrumpir la sesión si faltan las condiciones necesarias para su buen desarrollo, tampoco habiendo obligación de redactar un acuerdo ilegal o inexequible». Está estrechamente relacionado con el quinto principio, el del respeto al orden público y a las leyes vigentes, ya mencionado anteriormente.

El sexto principio es el del empoderamiento, definido como el «deber de estimular a los interesados a aprender a resolver de la mejor manera sus futuros conflictos, en función de la experiencia de justicia vivida en la autocomposición» (artículo 1, VI). Este principio está unido al séptimo principio, el de la validación, que corresponde al «deber de estimular a los interesados a percibirse recíprocamente como seres humanos merecedores de atención y respeto» (artículo 1, VII). Así, se ratifica la conveniencia absoluta de utilizar la mediación para el tratamiento de conflictos laborales colectivos. Considerando que el oficio del mediador consiste en buscar restaurar la capacidad de diálogo entre las partes conflictivas, el éxito en el cumplimiento de ese menester posee la innegable propiedad de prevenir futuros conflictos, evitando la reiteración de situaciones extremas como la huelga:

En el examen de la mediación/conciliación es importante recordar que, aunque participe un tercero, representan mecanismo fundamental para que las partes adquieran madurez en el ejercicio de la ciudadanía porque, en última instancia, son ellas las que construyen la solución para el conflicto.

10. «A imparcialidade do mediador radica na equidistância face às partes mediadas: o mediador não pode aliar-se a uma das partes ou tomar um partido, não obstante os previsíveis esforços dos mediados em cativar a sua preferencia» (Lopes y Patrão, 2016: 55). 
Se tiene, en consecuencia, el empoderamiento de las partes como resultado de su desempeño en el proceso de mediación/conciliación, así como la denominada validación, en la que las partes pasan a distinguir el otro como alguien que merece respeto y consideración (Koury, 2016: 133). ${ }^{11}$

Los principios que orientan la actuación de mediadores en la justicia del trabajo son los mismos adoptados para la mediación judicial en general en Brasil, los cuales se encuentran listados en el Código de Ética de Conciliadores y Mediadores Judiciales (Anexo III de la Resolución CNJ 125/2010). Sin embargo, la Resolución CSJT 174/2016 parece haber olvidado el principio de la confidencialidad, correspondiente al deber de mantener secreto sobre todas las informaciones obtenidas en la sesión, salvo autorización expresa de las partes o violación al orden público o a las leyes vigentes, no pudiendo ser testigo del caso, ni actuar como abogado de los involucrados, en cualquier hipótesis (artículo 1, I, del Anexo III de la Resolución CNJ 125/2010). Según Gouveia (2019: 98), la confidencialidad es esencial para que haya plena confianza en el proceso de mediación.

Ante la omisión de un principio fundamental de la mediación (no por casualidad, el primero de su lista), cabe indagar qué razón habría llevado el CSJT a no insertarlo en la Resolución 174/2016:

El artículo 1 del Código de Ética de la Resolución 174 del CSJT no enumera la confidencialidad como principio de la mediación. Tal vez en función del tipo de disputa allí tratada, ese no fue considerado un principio importante. Sin embargo, excluir la confidencialidad puede herir de muerte cualquier procedimiento de mediación. Para que el mediador gane la confianza de las partes, necesita garantizar de que lo que se diga o se proponga no salga de la sala de reuniones. Difícilmente las personas hablarán sobre la disputa de manera abierta y sincera si temen que el relato pueda ser utilizado por la otra parte para obtener ventajas o entonces que pueda ser utilizado como argumento para procedencia o no del pedido. Así, excluir la confidencialidad es herir de muerte la mediación, es crear un nuevo procedimiento que pueda alcanzar el consenso, pero que no podrá ser llamado de procedimiento de mediación (Spengler, 2017: 141)..12

11. «No exame da mediação/conciliação é importante lembrar que, embora delas participe um terceiro, representam mecanismo fundamental para que as partes adquiram maturidade no exercício da cidadania porquanto, em última análise, são elas que constroem a solução para o conflito.

TEM-SE, com consequência, o empoderamento das partes em decorrência de sua atuação no processo de mediação/conciliação, como também a chamada validação, em que as partes passam a distinguir o outro como alguém merecedor de respeito e consideração «En el examen de la mediación/conciliación es importante recordar que, aunque participe un tercero, representan mecanismo fundamental para que las partes adquieran madurez en el ejercicio de la ciudadanía porque, en última instancia, son ellas las que construyen la solución para el conflicto» (Koury, 2016: 133).

12. «Ao art. $1^{\circ}$ do Código de Ética da Resolução 174 do CSJT não elenca a confidencialidade como 
Por último, entre las reglas que orientan el procedimiento de mediación (dispuestas en el artículo 2 del Anexo II de la Resolución CSJT 174/2016), se encuentran: el deber de información (según el cual el mediador debe aclarar a los involucrados el método de trabajo a ser adoptado, las normas de conducta y las fases del procedimiento); la autonomía de la voluntad de las partes (las cuales deben llegar a un acuerdo de modo espontáneo, sin ningún tipo de coerción sobre su albedrío); la ausencia de obligación de resultado (pues, como ya se ha expuesto, el éxito de una mediación no está directamente relacionado a la obtención de un acuerdo, sino a la restauración del diálogo entre los implicados); la desvinculación del mediador de su profesión de origen (porque el mediador debe hacer esfuerzos para desprenderse psicológicamente de su oficio originario - ya sea de magistrado o de servidor- durante la mediación); y la comprensión de las partes en cuanto a la mediación (a fin de que ellas sepan exactamente cuáles son las implicaciones del acuerdo, comprometiéndose firmemente a respetar los términos convenidos).

\section{Conclusión}

Las relaciones laborales, a pesar de encontrarse, por regla general, en un estado de equilibrio, contienen de modo subyacente un conflicto latente, resultado de las tensiones derivadas de la divergencia natural de intereses entre capital y trabajo.

En el Poder Judicial brasileño, la Política Judicial Nacional de tratamiento adecuado de los conflictos de intereses está regulada, en general, por la Resolución CNJ 125/2010.

En la justicia del trabajo brasileña, esa política es objeto de la Resolución CSJT 174/2016, la cual dispone, entre otros, sobre el instituto de la mediación.

La mediación se caracteriza como uno de los más relevantes métodos autocompositivos y no adversariales de tratamiento de conflictos, pudiendo ser adoptada tanto en la fase preprocesal como en la fase procesal.

Para el tratamiento de conflictos de naturaleza colectiva en el ámbito de la justicia del trabajo brasileña, la mediación procesal es un método altamente recomendable, pues busca restablecer la capacidad de diálogo entre las partes. Sin embargo, es esencial que los magistrados y servidores que se dispongan a oficiar como mediadores

princípio da mediação. Talvez em função do tipo de disputa ali tratada esse não foi considerado um princípio importante. Porém, excluir a confidencialidade fere de morte qualquer procedimento de mediação. Para que o mediador ganhe a confiança das partes, ele precisa garantir a elas que o que for dito ou proposto não sairá da sala de reunião. Dificilmente as pessoas vão falar sobre a disputa de modo aberto e sincero se temerem que o relato possa ser usado pela outra parte para obter vantagens ou então que possa ser utilizado como argumento para procedência ou não do pedido. Assim, excluir a confidencialidade é ferir de morte a mediação, é criar um novo procedimento que pode alcançar o consenso, mas que não poderá ser chamado de mediativo» (Spengler, 2017: 141). 
obedezcan rigurosamente a los principios y reglas constantes en el Código de Ética de Conciliadores y Mediadores Judiciales, bajo pena de desvirtuar la finalidad del instituto.

\section{Referencias}

Almeida, Mario de (2016). «Abordaje de conflictos laborales». En Walter A. Wright (director), María Alba Aiello y Mario de Almeida (coordinadores), Abordaje de conflictos. Ciudad Autónoma de Buenos Aires: Astrea.

ArLÉ, Danielle de Guimarães Germano (2016). Mediação, negociação e práticas restaurativas no Ministério Público. Belo Horizonte: Editora D’Plácido.

Azócar Simonet, Rodrigo y Álvaro Cruz González (2015). «Limitaciones al derecho de huelga en Chile: Los servicios esenciales, el reemplazo de trabajadores y los servicios mínimos en el contexto de la Reforma Laboral». Revista Chilena de Derecho del Trabajo y de la Seguridad Social, 6 (12): 140-161. Disponible en «http:// bit.ly/2I9LX77».

Felipe, Ana Paula Faria y Manuela Duarte Almeida Pinto (2018). «Administração de conflitos no âmbito da saúde». En Fernanda Duarte, Rafael Mario Iorio Filho, Ana Paula Faria Felipe, Delton Meirelles (coordinadores), Escritos sobre direito, cidadania e processos: discursos e práticas. Niterói: PPGSD, Programa de Pós-Graduação em Sociologia e Direito.

Freund, Julien (1995). Il terzo, il nemico, il conflitto: materiali per una teoria del Politico. Milano: Giuffrè.

Fuenzalida Martínez, Patricia (2015). «Judicialización del conflicto colectivo y el rol del juez en su solución». Revista Chilena de Derecho del Trabajo y de la Seguridad Social, 6 (12): 82-101. Disponible en http://bit.ly/2KjFA3Y.

Gouveia, Mariana França (2019). Curso de resolução alternativa de litígios. Coimbra: Almedina.

GunTHER, Luiz Eduardo (2016). «A aplicabilidade do instituto da mediação aos conflitos trabalhistas». En Cláudia Maria Gomes de Souza e outros (coordinadores), Mediação de conflitos: a emergência de um novo paradigma. Belo Horizonte: Del Rey.

KouRY, Luiz Ronan Neves (2016). «Mediação e conciliação no novo código de processo civil - seus desdobramentos no direito processual do trabalho». En Cláudia Maria Gomes de Souza e outros (coordinadores), Mediação de conflitos: a emergência de um novo paradigma. Belo Horizonte: Del Rey.

LoPes, Dulce y Afonso Patrão (2016). Lei da mediação comentada. Coimbra: Almedina.

Morales, Hugo Ítalo (2011). «La conciliación laboral». Revista Chilena de Derecho del Trabajo y de la Seguridad Social, 2 (3): 199-205. Disponible en http://bit. ly/2Zoops2. 
Muñoz, Pascual Ortuño (2018). Justicia sin jueces: métodos alternativos a la justicia tradicional. Barcelona: Ariel.

Nascimento, Amauri Mascaro y Sônia Mascaro Nascimento (2018). Iniciação ao direito do trabalho. São Paulo: LTr.

Pereira, Rita Andréa Guimarães de Carvalho (2016). «Mediação: o eco do vivido e o prelúdio do vir a ser. O papel do instituto em relação aos chamados métodos de resolução de conflitos». En Cláudia Maria Gomes de Souza e outros (coordinadores), Mediação de conflitos: a emergência de um novo paradigma. Belo Horizonte: Del Rey.

Pinho, Humberto Dalla Bernardina de y Matheus Sousa Ramalho (2017). «A mediação como ferramenta de pacificação de conflitos». Revista dos Tribunais, 975. Disponible en: «http://bit.ly/2QF6Nzg».

Regla, Josep Aguiló (2015). El arte de la mediación: argumentación, negociación y mediación. Madrid: Trotta.

Silva, De Plácido e (2009). Vocabulário jurídico. Actualizadores: Nagib Slaibi Filho y Gláucia Carvalho. Río de Janeiro: Forense.

Spengler, Fabiana Marion (2016). Da jurisdição à mediação de conflitos: por uma outra cultura no tratamento de conflitos. Ijuí: Ed. Unijuí.

-. (2017). MEdiaÇão de CONFlitos: da teoria À PRÁtica. Porto Alegre: LivraRIA DO ADVOGado.

Spengler, Fabiana Marion y Theobaldo Spengler Neto (2008). «O 'tempo da jurisdição' e o 'tempo da mediação': a(s) verdade(s) conflitiva(s) e o seu tratamento». Revista do Direito, Santa Cruz do Sul, 2008: 99-118. Disponible en: «http://bit. ly/2JQ5JId».

TARTUCE, Fernanda (2018). Mediação nos conflitos civis. Río de Janeiro: Forense; São Paulo: Método.

\section{Sobre los autores}

Fabiana Marion Spengler es posdoctora en Derecho por la Università degli Studi di Roma Tre, en Italia. Doctora en Derecho por la Universidade do Vale do Rio dos Sinos, Brasil. Magíster en Desarrollo Regional por la Universidade de Santa Cruz do Sul (UNISC), en Brasil. Docente de los cursos de Grado y Postgrado lato y stricto sensu de UNISC. Coordinadora del Grupo Investigador «Políticas Públicas no Tratamento dos Conflitos», vinculado al CNPq (Consejo Nacional de Desarrollo Científico y Tecnológico de Brasil). Autora de diversos libros y artículos científicos. Correo electrónico: fabiana@unisc.br.

Márcio Dutra Da Costa es Alumno de Máster en Derecho por la Universidade de Santa Cruz do Sul, en Brasil. Especialista en Derecho y Proceso Laboral por la Universidade para o Desenvolvimento do Estado e da Região do Pantanal, Brasil. 
Miembro del Grupo Investigador «Políticas Públicas no Tratamento dos Conflitos», vinculado al CNPq (Consejo Nacional de Desarrollo Científico y Tecnológico de Brasil). Fiscal laboral (Ministerio Público del Trabajo). Correo electrónico: marciodc@ hotmail.com. 
La Revista Chilena de Derecho del Trabajo y de la Seguridad Social es una publicación semestral del Departamento de Derecho del Trabajo y de la Seguridad Social de la Facultad de Derecho de la Universidad de Chile, y que tiene por objetivo el análisis dogmático y científico de las instituciones jurídico-laborales y de seguridad social tanto nacionales como del derecho comparado y sus principales efectos en las sociedades en las que rigen.

\author{
DIRECTOR \\ Luis Lizama Portal \\ EDITOR \\ Claudio Palavecino Cáceres \\ SECRETARIO DE REDACCIÓN \\ Eduardo Yañez Monje \\ SITIO WEB \\ revistatrabajo.uchile.cl \\ CORREO ELECTRÓNICO \\ pyanez@derecho.uchile.cl \\ LICENCIA DE ESTE ARTÍCULO \\ Creative Commons Atribución Compartir Igual 4.o Internacional
}

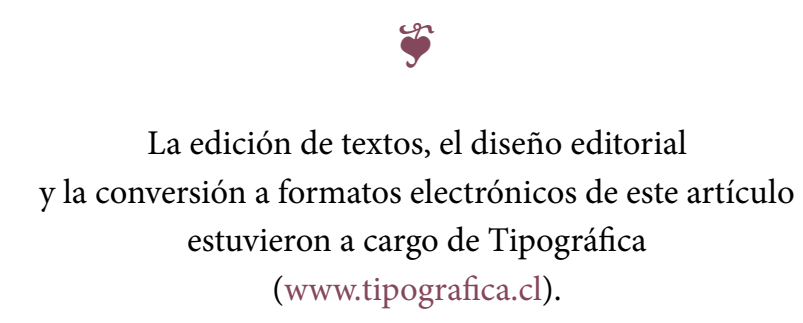

